\title{
367. Bauchdeckenersatz bei der Ratte durch Dura
}

\author{
H. H. Werner, W. Izbicki und H. Günther \\ 2. Lehrstuhl für Chirurgie, Köln-Merheim, Pathologisches Institut der Universität Köln, \\ Ostmerheimer Straße 200, D-5000 Köln 91
}

\section{Replacement of the Abdominal Wall in the Rat by Dura}

Summary. In a randomized study in 20 male Wistar rats a defect in the abdominal wall, $3 \times 4 \mathrm{~cm}$ in size, was covered wit Lyodura and Lyodura soft. Eleven transplants $(55 \%)$ showed primary healing. One animal died as a result of infection and a burst abdomen. The animals were killed after 3 months. The tensile strength of the wounds treated with Lyodura soft and showing primary healing was three times that of the wounds closed with normal dura (median: $10.9 \mathrm{~N} / 3.6 \mathrm{~N}$ ). Both kinds have proved good histological compatibility, Lyodura soft being integrated more slowly.

Key words: Replacement of wall dura - Compatibility - Tensile strength.

Zusammenfassung. Bei 20 männlichen Wistar-Ratten wurde ein Bauchwanddefekt der Größe $3 \times 4 \mathrm{~cm}$ nach Randomisierung durch Lyodura und Lyodura ,soft" gedeckt. 11 Transplantate $(55 \%)$ heilten primär ein. Ein Tier verstarb durch Infektion und Platzbauch. Die Tötung der Tiere erfolgte nach 3 Monaten. Die Reißfestigkeit der mit Lyodura soft behandelten Wunden mit Primärheilung war dreimal größer als die mit normaler Dura verschlossenen (Median: 10,9 N/3,6 N). Beide Sorten erwiesen sich histologisch als gut verträglich. Lyodura soft wird langsamer umgebaut.

Schlïsselwörter: Bauchdeckenersatz - Dura - Verträglichkeit - Reißfestigkeit.

\section{Standardisierte mikrochirurgische Gefäßtechnik - End/End und End/Seit-Anastomosen in der experimentellen Transplantationschirurgie}

\author{
G. H. Müller, U. T. Hopt, J. Gale ${ }^{1}$ und P. J. Morris ${ }^{1}$ \\ Chirurgische Universitätsklinik Tübingen, Calwer Straße 7, D-7400 Tübingen, ${ }^{1}$ Nuffield Department of \\ Surgery, Oxford, University, GB

\section{Standardized Technik in Microvascular Surgery - End-to-End and End-to-Side-Anastomoses for Experimental Organ Transplantation}

Summary. Heart, kidney, spleen, pancreas and liver grafts have been performed using four different techniques for vascular anastomoses: "end-to-end" interrupted sutures and continuous sutures. "End-to-side" interrupted sutures and continuous sutures. End-to-end seems to be the safer technique, end-to-side to be technically easier. Continuous sutures are the choice for venous anastomoses. These techniques can be recommended for experimental as well as clinical use.

Key words: Transplantation - Techniques - Microsurgery.

Zusammenfassung. Herz-, Nieren-, Milz-, Pankreas- und Lebertransplantate wurden mit 4 verschiedenen Gefäßanastomosen ausgeführt. End-zu-End-Einzelkopfnaht - fortlaufende Naht. End-zu-Seit-Einzelkopfnaht - fortlaufende Naht. End-zu-End-Anastomosen erschienen insgesamt sicherer, End-zu-Seit technisch einfacher. Fortlaufende Nähte haben sich besonders an venösen Anastomosen bewährt. Die beschriebenen Techniken werden für die experimentelle und klinische Anwendung empfohlen.

Schlïsselwörter: Transplantation - Technik - Mikrochirurgie. 